\title{
Adaptabilidad a las tecnologías de la información, comunicación y procrastinación laboral en colaboradores del sector público en Arequipa
}

Fecha de recepción : 2020-08-21 • Fecha de aceptación: 2020-11-10 • Fecha de publicación: 2021-10-01

José Luis Arias Gonzáles

Enfoques Consulting

Joseariasgon6@gmail.com

https://orcid.org/0000-0002-3250-5287

\section{Resumen}

El presente artículo científico tuvo como objetivo principal establecer la relación entre la adaptabilidad a las Tecnologías de la Información y Comunicación (TIC) y la procrastinación laboral en colaboradores del sector público en Arequipa, se desarrolló bajo los lineamientos metodológicos de una investigación básica, correlacional y no experimental-transversal, se utilizó como técnica la encuesta y como instrumentos, el cuestionario Ce-PAC y la escala PAWS, la recolección de datos se hizo de forma virtual por medio de formularios de Google; la población se conformó por 18,589 colaboradores y para hallar la muestra se utilizó el método probabilístico-aleatorio simple, quedando conformada por 376 colaboradores. Los principales resultados demostraron que el nivel de adaptabilidad a las TIC de los colaboradores del sector público fue baja y en consecuencia, el nivel de procrastinación laboral alta, lo que resulta una relación significativa inversa, la cual fue demostrada mediante la prueba de hipótesis de Chi cuadrado con un nivel de significancia de 0.000 , asimismo, se evidenció que la mayoría de colaboradores tienen más de 41 años de edad, y que las mujeres tienen mayor nivel de adaptabilidad a las TIC y menor procrastinación laboral que los hombres. 


\begin{abstract}
The main aim of this scientific article was to establish the relationship between adaptability to Information and Communication Technologies (ICT) and labor procrastination in public sector employees in Arequipa, this research was developed under the methodological guidelines of a basic correlational and non-experimental-transversal investigation, the survey was used as a technique, and as instruments the Ce-PAC questionnaire and the PAWS scale, the data collection was done virtually through Google forms; the population made up of 18,589 employees, and to find the sample the simple probabilistic-random method was used, it was made up of 376 collaborators. The main results showed that the level of adaptability to ICT of public sector employees was low and, consequently the level of labor procrastination was high, resulting in a significant inverse relationship, which was demonstrated by the Chi square hypothesis test with a significance level of 0.000 , likewise, it was evidenced that the majority of employees are over 41 years old, and that women have a higher level of adaptability to ICT and less labor procrastination than men.
\end{abstract}

Keywords: information and Communication Technologies, technological tools, labor procrastination, public sector, adaptability. 


\section{Introducción}

La tecnología, hoy en día está presente en la mayoría de ámbitos de la vida de los seres humanos, y es necesario mencionar que se ha convertido en un elemento para el desarrollo laboral y económico de las organizaciones, asimismo, se puede notar las facilidades que la tecnología brinda para crear riquezas, facilitar los procesos educativos y empresariales, simplificar la búsqueda de información, comunicarse con gente de otros lugares de forma inmediata, optimizar tiempos en el trabajo, entre otros beneficios ofrecidos por las Tecnologías de la Información y Comunicación (TIC); sin embargo, si bien, para la mayoría resulta una herramienta que les facilita la vida y el trabajo, para algunos el acceso y uso de las TIC se ha vuelto una situación difícil, en especial para un segmento de personas que se encuentran entre los 40 y 65 años de edad, y llegado a este punto se puede presentar como un serio problema dado que, estas personas aún no se han identificado plenamente con los avances tecnológicos (Casamayou y Morales, 2017).

De acuerdo con Cobo (2009), las Tecnologías de la Información y Comunicación (TIC), se refieren a las redes informáticas que permiten la interacción de los ordenadores y el procesamiento de la información, además de interactuar de forma remota entre personas de distintos lugares, según Cano-Pita (2018), estas son importantes para mejorar el desempeño y la comunicación de la empresa, aunque se deben aplicar de forma inteligente y consciente, mientras que para Thompson y Strickland (2012), son dispositivos, herramientas, componentes electrónicos y equipos que adecuan la información y posibilitan el desarrollo de cualquier organización; existen múltiples medios de TIC como los celulares, los correos, los servidores y las plataformas digitales las cuales permiten intercambiar y acceder a la información desde cualquier parte del mundo.

Del bono y Henry (2008) precisan que las TIC han influenciado de forma directa en la forma de comunicación y actuación, además de la forma de trabajar y un medio para utilizar las TIC en el ámbito laboral es el teletrabajo, al tiempo que Del bono y Osio (2011), lo definen como una sustitución del lugar que esta relacionado con la actividad laboral y teniendo como princnipal herramienta las tecnologías de la información y comunicación de tal forma que se posibilite enviar el trabajo al trabajador en vez de el trabajador al trabajo.

Las TIC han cambiado la forma de trabajar y gestionar las actividades laborales; en un sentido positivo, ayudan a agilizar la comunicación, gestionar eficientemente los procesos empresariales y obtener información rápida y confiable, y desde un sentido negativo puedan llegar a causar frustración o abandono de las actividades laborales por la falta de conocimiento o adaptación para su uso.

El Sector público en Perú está constituido por tres poderes: Ejecutivo, legislativo y judicial, los cuales están al servicio de cada uno de los ciudadanos peruanos. Desde el mes de marzo del año 2020 todas las organizaciones del sector público están realizando sus actividades laborales de forma remota utilizando Tecnologías de la Información y Comunicación (TIC) como Zoom, Google Meet, Webinar, formularios de Google, entre otros, y se pronostica que en adelante las organizaciones utilizarán estas herramientas para la mayoría de sus actividades laborales. 
Por otro lado, desde la fecha mencionada, según los reportes de algunas organizaciones, se están presentando con mayor continuidad de forma tardía las tareas y actividades encomendadas a los colaboradores, y en algunos casos, se demoran hasta 2 o 3 días, por tal razón, el presente artículo científico tiene como objetivo principal establecer la relación entre la adaptabilidad a las Tecnologías de la Información y Comunicación (TIC) y la procrastinación laboral en colaboradores del sector público en Arequipa.

\section{Adaptabilidad}

La adaptabilidad se ha estudiado desde varias áreas de la vida de los individuos y la sociedad, en este caso se expone desde el ámbito laboral, donde en los últimos años se han presentado cambios disruptivos en las organizaciones como las nuevas formas de trabajo online, remotos o virtuales, o el uso de recursos tecnológicos y las herramientas para la gestión de las organizaciones.

Según Arnau (2011), la adaptabilidad es la capacidad que tiene un individuo para afrontar cualquier cambio en su entorno laboral que siempre es cambiante, esta capacidad se muestra de forma proactiva afrontando las exigencias en el puesto de trabajo.; la adaptabilidad permite incrementar el grado de sincronización del trabajador con el trabajo, alineandose a las necesidades de la empresa y los requerimientos de su puesto de trabajo. Según Garzón (2018), cuando un individuo se adapta, responde de forma positiva a las condiciones cambiantes y está determinado por esa capacidad de aprender y responder a las conjeturas del entorno.

\subsection{Adaptabilidad a las Tecnologías de la Información y Comunicación}

La incorporación de las TIC han producido consecuencias positivas y negativas en la sociedad, y de forma directa en las actividades laborales de los individuos, muchas veces de forma obligada y otras para optimizar los procesos en las organizaciones como empresas del sector público y privado o instituciones educativas, por lo cual, los colaboradores deben optar por aquellas herramientas tecnológicas que facilitan el trabajo o les permiten una continuidad en sus labores, en ese sentido, las organizaciones no solo se enfrentan a un cambio tecnológico, sino también, al cambio sociolaboral que este puede producir en los colaboradores y su forma de adaptarse a los medios tecnológicos.

Cuando se hace referencia a la adaptación en las TIC se debe pensar en "cambio" (Phillips, 1995), es en ese momento donde se debe establecer los aspectos que se verán afectados y las situaciones que deben ser adaptadas como: las rutinas organizativas, cambios en los horarios y formas de trabajo, cambios en las formas para gestionar la información y transmitirlas, todo ello mediante nuevas formas vinculadas a las TIC.

Arnau (2011) refiere tres dimensiones que se pueden medir en la adaptabilidad, estas son: la cognitiva, que se manifiesta mediante la asociación del desarrollo de las competencias y habilidades que tiene el individuo para hacer frente al cambio tecnológico; la dimensión conductual, que se expresa mediante las experiencias previas, los hábitos y la actitud que muestra el individuo frente al cambio tecnológico, por último, la dimensión emocional, que consiste en la 
adaptación por medio del entorno laboral, las relaciones laborales y la participación que muestra el individuo para lograr adaptarse al cambio (Fraga, 2014).

Un estudio realizado por Cano-Pita (2018) muestra que las organizacines deben ir de la mano con la evolución de la sociedad, los avances tecnológicos y la globalización, además, es importante para el desarrollo de las empresas que el personal protagonice dicho cambio y se adapte. Porter (2000) precisa que el cambio tecnológico es fundamental para la ventaja competitiva; sin embargo, esto no quiere decir que sea beneficioso, en algunos sectores puede empeorar el desempeño de las empresas por la falta de adaptación de los colaboradores. Por otro lado, los resultados obtenidos por Hernández (2017), muestran que lo que más afectó la incorporación de las TIC a las empresas fue la falta de adaptabilidad de los individuos; pero a esto, al transcurrir el tiempo, la sociedad depende del enfoque tencológico, por lo que es imprescindible la adaptación.

\section{Procrastinación laboral}

Existen colaboradores que envían tarde una tarea encomendada por su supervisor o realizan sus actividades en un tiempo mayor al establecido, esta situación siempre se observa en las organizaciones del sector público; sin embargo, llega un momento en el que se hace repetitiva y en mayor grado, por lo que se muestra preocupante para el desarrollo de la organización, y se vuelve un esquema procrastinador.

Para los autores Hernández y García (2018), la procrastinación es un hábito que perdura en el tiempo, en lugar de afrontar las responsabilidades, se crean excusas para postergar el trabajo, idea similar a la de García (2011), quien manifiesta que, la procrastinación laboral se trata de postergar de forma sistemática las tareas laborales que son relevantes para el desarrollo de nuestra actividad, postergar implica demorar el inicio de una tarea obligatoria o aplazarla para llevar a cabo otras actividades que no tengan nada que ver con el trabajo.

Estudios realizados por Sobalvarro (2008) muestran que, la capacidad adaptativa es importante para el desempeño de los trabajadores porque les permite realizar sus tareas sin demora y afrontando las actividades diarias según lo establecido.

\section{Metodología}

Los lineamientos metodológicos que se utilizaron para el artículo científico corresponden a una investigación básica, según Hernández-Sampieri y Mendoza, (2018), se caracteriza por no tener fines prácticos de forma inmediata, su principal objetivo es incrementar la teoría y principios fundamentales con respecto a sus variables de estudio.

El alcance de investigación utilizado fue el descriptivo-correlacional, este tipo de estudios se fundamentan en la descripción de sus variables de estudio o fenómenos para asociarlos o relacionarlos entre sí (Hernández -Sampieri y Mendoza, 2018). 
Por último, el diseño fue no experimental de corte transversal, según Hernández-Sampieri y Mendoza (2018), este diseño se establece cuando no se van a manipular las variables de estudio y se realiza en un solo periodo de tiempo.

La población estuvo constituida por 18,589 colaboradores del sector público de la ciudad de Arequipa, y para obtener la muestra se utilizó el método de muestreo probabilístico - aleatorio simple, que consiste en tomar a un sugbrupo representativo de la población con personas con las mismas probabilidades de ser elegidas (Hernández -Sampieri y Mendoza, 2018); la muestra se conformó por 376 colaboradores del sector público.

La técnica que se utilizó para ambas variables fue la encuesta, el instrumento utilizado para la variable adaptabilidad a las TIC fue el cuestionario de Ce-PAC, y el instrumento utilizado para la variable procrastinación laboral fue la Eescala de procrastinación en el trabajo (PAWS), ambos con una codificación de: totalmente de acuerdo, (5), de acuerdo (4), indiferente (3), en desacuerdo (2) y totalmente en desacuerdo (1); la calificación fue de alta, regular, baja y nula para ambas variables.

Para la recolección de los datos se utilizó el formulario de Google en el que se crearon las preguntas y respuestas para enviárselas a los participantes del estudio por medio de un link a sus correos electrónicos; las respuestas obtenidas fueron evaluadas, contabilizadas y codificadas para corroborar que no falte ninguna y que no haya información mal redactada, una vez hecho esto, se sistematizaron las respuestas en el Software Excel 2016 y se generaron las tablas de distribución de frecuencias; posterior a ello, se utilizó el Software IBM SPSS stadistic 25 para aplicar la prueba de normalidad bajo los parámetros de Kolmorogov-Smirnov y se identificó el tipo de variables (Cuantitativas o categóricas) para establecer la prueba de hipótesis a utilizar que fue Chi cuadrado con un nivel de confianza del $95 \%$.

- La hipótesis de investigación fue $(\mathrm{H} 1)$ : existe relación significativa entre la adaptabilidad a las tecnologías de la información y comunicación (TIC) y la procrastinación laboral en colaboradores del sector público en Arequipa.

- La hipótesis nula fue (Ho): no existe relación significativa entre la adaptabilidad a las tecnologías de la información y comunicación (TIC) y la procrastinación laboral en colaboradores del sector público en Arequipa.

\section{Resultados}

Para presentar los resultados se tomaron en cuenta datos sociodemográficos como la edad y el género de los colaboradores del sector público, luego, se analizaron las variables de estudio mediante las tablas de distribución de frecuencias, por último, se presentó la prueba de hipótesis.

Es importante, previamente, conocer la edad y el género de los colaboradores para diferenciar que segmento presenta mayor o menor nivel de adaptabilidad a las TIC y procrastinación laboral. 
Tabla 1.

Segmentos por edades de los colaboradores objeto de estudio

\begin{tabular}{|l|l|l|}
\hline Edad & Frecuencia & Porcentaje \\
\hline $18-40$ & 42 & $11 \%$ \\
\hline $41-55$ & 162 & $43 \%$ \\
\hline 56 a más & 172 & $46 \%$ \\
\hline
\end{tabular}

Fuente: elaboración propia en base a los resultados del estudio

En la Tabla 1 se observa que el $89 \%$ de colaboradores del sector público tienen más de 41 año de edad, y sólo el $11 \%$ tienen entre 18 y 40 años, en su mayoría, los trabajadores con más de 41 años de edad son nombrados, es decir, llevan trabajando más de 5 años en el sector público.

Tabla 2.

Género de los colaboradores objeto del estudio

\begin{tabular}{|l|l|l|}
\hline Género & Frecuencia & Porcentaje \\
\hline Hombre & 209 & $56 \%$ \\
\hline Mujer & 167 & $44 \%$ \\
\hline
\end{tabular}

Fuente: elaboración propia en base a los resultados del estudio

En la Tabla 2, se muestra que, la cantidad de hombres es mayor a la cantidad de mujeres que laboran en el sector público, lo que evidencia una disparidad.

Tabla 3.

Nivel de adaptabilidad a las TIC según el género de los colaboradores

\begin{tabular}{|l|l|l|l|l|l|l|}
\hline & \multicolumn{4}{|l|}{ Hombre } & \multicolumn{3}{l|}{ Mujer } & TOTAL \\
\hline Nivel & Frecuencia & Porcentaje & Frecuencia & Porcentaje & Frecuencia & Porcentaje \\
\hline Alto & 6 & $2 \%$ & 29 & $7 \%$ & 35 & $9 \%$ \\
\hline Regular & 10 & $3 \%$ & 16 & $4 \%$ & 26 & $7 \%$ \\
\hline Bajo & 186 & $50 \%$ & 118 & $31 \%$ & 304 & $81 \%$ \\
\hline Nula & 7 & $2 \%$ & 4 & $1 \%$ & 11 & $3 \%$ \\
\hline
\end{tabular}

Fuente: elaboración propia en base a los resultados del estudio

En la Tabla 3 se evidencia que el nivel de adaptabilidad a las TIC del $81 \%$ de colaboradores del sector público es bajo, lo que representa a la mayoría de colaboradores, asimismo, el $50 \%$ de hombres y el $31 \%$ de mujeres presentan un nivel de adaptabilidad a las TIC baja, por tanto, los resultados permiten establecer que, del $81 \%$ de colaboradores con nivel alto de adaptabilidad a las $\mathrm{TIC}$, los hombres tienen mayor predominio en este nivel. 
Tabla 4.

Nivel de adaptabilidad a las TIC según la edad de los colaboradores

\begin{tabular}{|l|l|l|l|l|l|l|l|l|}
\hline & $\mathbf{1 8}-\mathbf{4 0}$ & $\mathbf{4 1}-\mathbf{5 5}$ & $\mathbf{5 6}$ a más & \multicolumn{2}{l|}{ TOTAL } \\
\hline Nivel & Frecuencia & Porcentaje & Frecuencia & Porcentaje & Frecuencia & Porcentaje & Frecuencia & Porcentaje \\
\hline Alta & 3 & $1 \%$ & 11 & $3 \%$ & 21 & $6 \%$ & 35 & $9 \%$ \\
\hline Regular & 3 & $1 \%$ & 16 & $4 \%$ & 7 & $2 \%$ & 26 & $7 \%$ \\
\hline Baja & 33 & $9 \%$ & 129 & $34 \%$ & 142 & $38 \%$ & 304 & $81 \%$ \\
\hline Nula & 3 & $1 \%$ & 6 & $2 \%$ & 2 & $1 \%$ & 11 & $3 \%$ \\
\hline
\end{tabular}

Fuente: elaboración propia en base a los resultados del estudio

En la Tabla 4 se observa que, los colaboradores del sector público con más de 56 años presentan predominio en el nivel bajo de adaptabilidad a las TIC (38\%), seguido del $34 \%$ de los colaboradores entre $41-55$ años de edad, por tanto, se infiere mediante los resultados que, del $89 \%$ de colaboradores con más de 41 años de edad, el $72 \%$ presentan un nivel bajo de adaptabilidad a las TIC.

Tabla 5.

Nivel de procrastinación laboral según el género de los colaboradores

\begin{tabular}{|l|c|c|c|c|c|c|}
\hline & \multicolumn{2}{|c|}{ Hombre } & \multicolumn{2}{c|}{ Mujer } & \multicolumn{2}{c|}{ TOTAL } \\
\hline Nivel & Frecuencia & Porcentaje & Frecuencia & Porcentaje & Frecuencia & Porcentaje \\
\hline Alta & 169 & $45 \%$ & 113 & $30 \%$ & 282 & $75 \%$ \\
\hline Regular & 14 & $4 \%$ & 25 & $6 \%$ & 39 & $10 \%$ \\
\hline Baja & 23 & $6 \%$ & 17 & $5 \%$ & 40 & $11 \%$ \\
\hline Nula & 3 & $1 \%$ & 12 & $3 \%$ & 15 & $4 \%$ \\
\hline
\end{tabular}

Fuente: elaboración propia en base a los resultados del estudio

En la Tabla 5 se visualiza que, el nivel de procrastinación laboral del $75 \%$ de colaboradores del sector público es alto, lo que representa las tres cuartas partes del total, asimismo, el $45 \%$ de hombres y el $30 \%$ de mujeres presentan un nivel de procrastinación laboral alta, en consecuencia, los resultados permiten establecer que, del $75 \%$ de colaboradores con nivel bajo de procrastinación laboral, los hombres tienen mayor predominio en este nivel. 
Tabla 6.

Nivel de procrastinación laboral según la edad de los colaboradores

\begin{tabular}{|l|c|c|c|c|c|c|c|c|}
\hline & \multicolumn{2}{|c|}{$\mathbf{1 8 - 4 0}$} & \multicolumn{2}{c|}{$\mathbf{4 1}-\mathbf{5 5}$} & \multicolumn{2}{c|}{56 a más } & \multicolumn{2}{c|}{ TOTAL } \\
\hline Nivel & $\begin{array}{c}\text { Frecuen- } \\
\text { cia }\end{array}$ & $\begin{array}{c}\text { Porcen- } \\
\text { taje }\end{array}$ & $\begin{array}{c}\text { Frecuen- } \\
\text { cia }\end{array}$ & $\begin{array}{c}\text { Porcen- } \\
\text { taje }\end{array}$ & $\begin{array}{c}\text { Frecuen- } \\
\text { cia }\end{array}$ & $\begin{array}{c}\text { Porcen- } \\
\text { taje }\end{array}$ & $\begin{array}{c}\text { Frecuen- } \\
\text { cia }\end{array}$ & $\begin{array}{c}\text { Porcen- } \\
\text { taje }\end{array}$ \\
\hline Alta & 12 & $3 \%$ & 119 & $32 \%$ & 151 & $40 \%$ & 282 & $75 \%$ \\
\hline $\begin{array}{l}\text { Regu- } \\
\text { lar }\end{array}$ & 4 & $1 \%$ & 21 & $6 \%$ & 11 & $3 \%$ & 36 & $10 \%$ \\
\hline Baja & 23 & $6 \%$ & 13 & $3 \%$ & 6 & $2 \%$ & 42 & $11 \%$ \\
\hline Nula & 3 & $1 \%$ & 9 & $2 \%$ & 4 & $1 \%$ & 16 & $4 \%$ \\
\hline
\end{tabular}

Fuente: elaboración propia en base a los resultados del estudio

Y en la Tabla 6 se puede ver que los colaboradores del sector público, con más de 56 años, presentan predominio en el nivel alto de procrastinación laboral (40\%), seguido del $32 \%$, de colaboradores entre $41-55$ años de edad, por lo cual, se infiere mediante los resultados que, del $89 \%$ de colaboradores con más de 41 años de edad, el $72 \%$ presentan un nivel alto de procrastinación laboral

\section{Prueba de hipótesis}

Previamente, para definir la prueba de hipótesis, se realizó la prueba de normalidad, que como resultado tuvo un nivel de significancia de 0.000 (Parámetros de Kolmorogov-Smirnov), lo que indica que los datos no tuvieron una distribución normal, a continuación, se presenta la tabla cruzada para presentar los resultados de las variables correlacionados.

Tabla 7.

Tabla cruzada de resultados de las variables de estudio

\begin{tabular}{|c|c|c|c|c|c|c|}
\hline & & & \multicolumn{3}{|c|}{ Procrastinación laboral } & \multirow{2}{*}{ Total } \\
\hline & & & Alta & Baja & Regular & \\
\hline \multirow{6}{*}{ Adaptabilidad a las TIC } & \multirow{2}{*}{ Baja } & Recuento & 42 & 39 & 225 & 306 \\
\hline & & $\%$ del total & $11 \%$ & $10 \%$ & $60 \%$ & $81 \%$ \\
\hline & \multirow{2}{*}{ Regular } & Recuento & 0 & 0 & 28 & 28 \\
\hline & & $\%$ del total & $0 \%$ & $0 \%$ & $7 \%$ & $7 \%$ \\
\hline & \multirow{2}{*}{ Alta } & Recuento & 0 & 0 & 42 & 42 \\
\hline & & $\%$ del total & $0 \%$ & $0 \%$ & $11 \%$ & $11 \%$ \\
\hline \multirow{2}{*}{\multicolumn{2}{|c|}{$\begin{array}{l}\text { Total } \\
\% \text { del total }\end{array}$}} & Recuento & 42 & 39 & 295 & 376 \\
\hline & & $11 \%$ & $10 \%$ & $79 \%$ & $100 \%$ & \\
\hline
\end{tabular}

Fuente: datos obtenidos de los resultados del estudio y sistematizados en IBM SPSS Stadistic 25

En la Tabla 7 se evidencian los resultados de las dos variables del estudio, en el cual se observa que el $60 \%$ de colaboradores del sector público tienen un nivel de adaptabilidad a las TIC bajo y 
un nivel de procrastinación laboral alto, esta tendencia evidencia que existe una relación inversa y proporcional entre las variables de estudio.

Tabla 8.

Prueba estadística para la prueba de hipótesis: Chi cuadrado

\begin{tabular}{|l|l|l|l|}
\hline & Valor & df & $\begin{array}{l}\text { Significación asintótica } \\
\text { (bilateral) }\end{array}$ \\
\hline Chi-cuadrado de Pearson & $23.617^{\mathrm{a}}$ & 4 & .000 \\
\hline Razón de verosimilitud & 38.147 & 4 & .000 \\
\hline Asociación lineal por lineal & 18.560 & 1 & .000 \\
\hline N de casos válidos & 376 & & \\
\hline
\end{tabular}

Fuente: datos obtenidos de los resultados del estudio y sistematizados en IBM SPSS Stadistic 25

Mientras que en la Tabla 8 se presentan los resultados de la prueba de hipótesis de correlación para las variables Adaptabilidad a las TIC y procrastinación laboral, los cuales muestran un valor de significancia de 0.000 , con este resultado se rechaza la Hipótesis nula: No existe relación significativa entre la adaptabilidad a las tecnologías de la información y comunicación (TIC) y la procrastinación laboral en colaboradores del sector público en Arequipa, y se acepta la Hipótesis de investigación: Existe relación significativa entre la adaptabilidad a las tecnologías de la información y comunicación (TIC) y la procrastinación laboral en colaboradores del sector público en Arequipa.

\subsection{Discusión de resultados}

La muestra se dividió en $56 \%$ de colaboradores del sector público hombres y el $44 \%$ mujeres, de los cuales, el $89 \%$ tiene más de 41 años de edad.

Los resultados establecieron que, el $81 \%$ de colaboradores del sector púbico presentaron un nivel bajo de adaptabilidad a las TIC, de los cuales, el 72\% tienen más de 41 años de edad, el 50\% son hombres y el $31 \%$ mujeres, por otro lado, el $75 \%$ presentaron un nivel alto de procrastinación laboral, de los cuales, el $45 \%$ son hombres y el $30 \%$ mujeres.

No se encontraron resultados que relacionen las variables Adaptabilidad a las TIC y procrastinación laboral; sin embargo, a continuación, se van a presentar resultados por cada variable de este estudio.

Los resultados obtenidos para la variable adaptabilidad a las TIC son similares a los presentados por García y Forero (2016), los investigadores demostraron que, la variable adaptabilidad se correlaciona con variables como el equilibrio laboral $(\mathrm{Rho}=0,584)$, la variable condiciones del ambiente de trabajo $(\mathrm{Rho}=0,76)$ y la variable naturaleza de la tarea $(\mathrm{Rho}=0,616)$. Cuentas (2017), en su estudio de la influencia de la adaptación socio laboral en la satisfacción y percepción de la productividad concluyó que, el $74.6 \%$ de colaboradores tuvieron un nivel bajo de adaptación, lo que influyó negativamente en la satisfacción laboral y la productividad. 
Resultados contrarios fueron obtenidos por Corona, Reyes y García (2012), en una muestra de participantes con edades de entre 40 y 63 años, de los cuales el $81.7 \%$ cuentan con la habilidad de adaptación, misma que les permite adecuarse a cualquier situación casi siempre.

Los resultados de la variable procrastinación laboral son similares a los de Campos (2020), quien demostró que, el nivel de procrastinación laboral del personal que labora en una entidad del estado es alto. Hernández y García (2018), evidenciaron en su estudio que la procrastinación laboral influye de forma sustancial y negativa en los procesos productivos de una organización.

\section{Conclusiones}

Se ha demostrado que, la mayoría de colaboradores tienen más de 41 años de edad, y que las mujeres tienen mayor nivel de adaptabilidad a las TIC y menor procrastinación laboral que los hombres; sin embargo, en términos generales, los colaboradores del sector público aún no se adaptan al uso de herramientas tecnológicas como Google Meet, Zoon, Webinar, ni a las aplicaciones para enviar documentos e informes como Formularios de Google o Wetransfer.

A su vez, se puedo concluir que los colaboradores muestran una actitud reacia a estos cambios tecnológicos, al cumplimiento de objetivos desde el trabajo remoto y se oponen al aprendizaje de las TIC; estos mismos trabajadores presentan sus tareas y actividades laborales en un horario fuera de lo establecido, evaden la responsabilidad por sus obligaciones como servidor público, no planifican sus horarios y actividades del día y eluden cualquier actividad que tenga que ver con el uso de la tecnología; estas situaciones podrían vulnerar el desarrollo de la organización. 


\section{Referencias}

Arnau, L. (2011). Cuestionario para evaluar la adaptabilidad de los adultos desempleados hacia los cambios profesionales: aplicación preliminar y características psicométricas. Revista electrónica de investigación educativa, 13(2), 99-112. https://redie.uabc.mx/redie/article/view/286

Campos, M. (2020). Relación entre la funcionalidad familiar y procrastinación laboral en una entidad del estado (Tesis de pregrado). Universidad Católica Los Ángeles Chimbote. Arequipa, Perú.

Cano-Pita, G. (2018). Las TICs en las empresas: evolución de la tecnología y cambio estructural en las organizaciones. Dominio de las ciencias, 4(1), 499-510. https://doi.org/10.23857/dc.v4i1.762

Casamayou, A., y Morales, M. (2017). Personas mayores y tecnologías digitales. Psicología, conocimiento y sociedad, 7(2), 199-226. https://doi.org/10.26864/PCS.v7.n2.9

Cobo, C. (2009). El concepto de tecnologías de la información. Benchmarking sobre las definiciones de las TIC en la sociedad del conocimiento. Revista de estudios de comunicación, 14(27), 295 - 318.

Corona, V., Reyes, J., y García, M. (2012). Adaptabilidad social laboral y actitudes hacia la jubilación. Asociación Nacional de Facultades y Escuelas de Contaduría y Administración, México. Obtenido de http://congreso. investiga.fca.unam.mx/docs/xvii/docs/E01.pdf

Cuentas, J. (2017). Influencia de la adaptación socio laboral en la satisfacción y percepción de la productividad del trabajo. (Tesis de pregrado). Universidad Nacional de San Agustín. Arequipa

Del bono, A., y Henry, L. (2008). La acción sindical en el marco de las formas globalizadas de producción. Reflexiones a partir de la expansión de los call centers de servicios de exportación en Argentina. Revista latinoamericana de estudios de trabajo (20), 7-31.

Del bono, Y., y Osio, L. (2011). Mujer, Cyberfeminismo y Teletrabajo. Revista Compendium, 13(24), 61-78.

Fraga, S. (2014). La adaptabilidad organizacional: un aporte conceptual para las organizaciones contemporáneas. Revista Española de Orientaciones y Psicopedagogía, 128-136. https://doi.org/10.5944/reop.vol.25. num.1.2014.12017

García, M., y Forero, C. (2016). Calidad de vida laboral y la disposición al cambio organizacional en funcionarios de empresas de la ciudad de Bogotá-Colombia. Acta Colombiana de Psicología, 19(1), 79-90. Obtenido de https://doi.org/10.14718/ACP.2016.19.1.5

García, M. (2011). Problemática multifactorial del absentismo laboral, el presentismo y la procrastinación en las estructuras en las que se desenvuelve el trabajador. (2011). Medicina y seguridad en el trabajo, 57(223), 111-120. https://doi.org/10.4321/S0465-546X2011000200004 
Garzón, M. (2018). Capacidad dinámica de adaptación. Revista científica "Visión del futuro", 22(1). Obtenido de https://www.redalyc.org/articulo.oa?id=357959311007

Hernández -Sampieri, R., y Mendoza, C. (2018). Metodología de la investigación. Las rutas cuantitativa, cualitativa y mixta. México: Mc Graw Hill. https://doi.org/10.17993/CcyLl.2018.15

Hernández, R. (2017). Impacto de las TIC en la educación: Retos y perspectivas. Revista Propósitos y representaciones, 5(1), 325-347. https://doi.org/10.20511/pyr2017.v5n1.149

Hernández, R., y García, T. (2018). Impacto de la procrastinación en la productividad del gerente. Revista Boletín Redipe, 7(11), 2020-228.

Phillips, N. (1995). Motivating for change: How to manage employee Stress. London: Pitman.

Porter, H. (2000). Ventaja competitiva. Creación y sostenimiento de un desempeño superior. México: Continental.

Sobalvarro, C. (2008). La toma de decisión y la procrastinación. Tesis de investigación. Guatemala: Universidad de San Carlos.

Thompson, A., y Strickland, M. (2012). Administración estratégica, teoría y casos (18va ed.). México D.F.: Mc Graw hill. 
Copyright (c) 2021 José Luis Arias Gonzáles

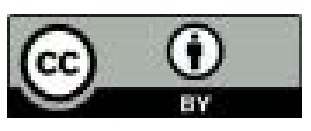

Este texto está protegido bajo una licencia internacional Creative Commons 4.0.

Usted es libre para Compartir-copiar y redistribuir el material en cualquier medio o formato - y Adaptar el documento - remezclar, transformar y crear a partir del material-para cualquier propósito, incluso para fines comerciales, siempre que cumpla las condiciones de Atribución. Usted debe dar crédito a la obra original de manera adecuada, proporcionar un enlace a la licencia, e indicar si se han realizado cambios. Puede hacerlo en cualquier forma razonable, pero no de forma tal que sugiera que tiene el apoyo del licenciante o lo recibe por el uso que hace de la obra.

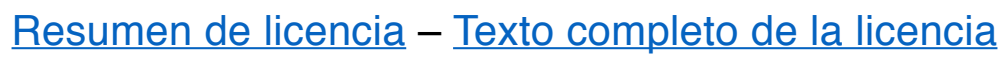

